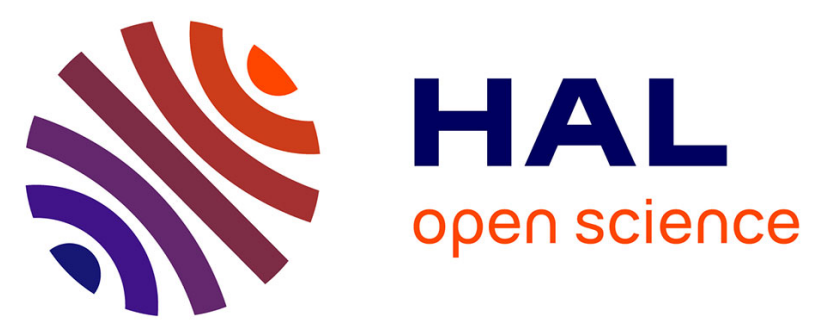

\title{
Non perturbative approach for a polar and polarizable linear molecule in an inhomogeneous electric field: Application to molecular beam deviation experiments
}

Emmanuel Benichou, Abdul-Rahman Allouche, Rodolphe Antoine, Monique Aubert-Frecon, Mickaël Bourgoin, Michel Broyer, Philippe Dugourd, Gerold Hadinger, Driss Rayane

\section{To cite this version:}

Emmanuel Benichou, Abdul-Rahman Allouche, Rodolphe Antoine, Monique Aubert-Frecon, Mickaël Bourgoin, et al.. Non perturbative approach for a polar and polarizable linear molecule in an inhomogeneous electric field: Application to molecular beam deviation experiments. The European Physical Journal D : Atomic, molecular, optical and plasma physics, 2000, 10 (2), pp.233-242. 10.1007/s100530050544. hal-00492376

\section{HAL Id: hal-00492376 https://hal.science/hal-00492376}

Submitted on 15 Jun 2010

HAL is a multi-disciplinary open access archive for the deposit and dissemination of scientific research documents, whether they are published or not. The documents may come from teaching and research institutions in France or abroad, or from public or private research centers.
L'archive ouverte pluridisciplinaire $\mathbf{H A L}$, est destinée au dépôt et à la diffusion de documents scientifiques de niveau recherche, publiés ou non, émanant des établissements d'enseignement et de recherche français ou étrangers, des laboratoires publics ou privés. 


\title{
Non perturbative approach for a polar and polarizable linear molecule in an inhomogeneous electric field: Application to molecular beam deviation experiments
}

\author{
E. Benichou a , A.R. Allouche, R. Antoine, M. Aubert-Frecon, M. Bourgoin, M. Broyer, Ph. Dugourd, \\ G. Hadinger, and D. Rayane
}

Laboratoire de Spectrométrie Ionique et Moléculaire ${ }^{\mathrm{b}}$, CNRS et Université Lyon I, bâtiment 205, 43 boulevard du 11 novembre 1918, 69622 Villeurbanne Cedex, France

Received 21 July 1999 and Received in final form 22 September 1999

\begin{abstract}
A non perturbative approach is used to solve the problem of a rigid linear molecule with both a permanent dipole moment and a static dipole polarizability, in a static electric field. Eigenenergies are obtained and compared to perturbative low field and high field approximations. Analytical expressions for the orientation parameters and for the gradient of the energy are given. This non perturbative approach is applied to the simulation of beam deviation experiments in strong electric field. Results of simulations are given for inhomogeneous alkali dimers. For LiNa, the simulations are compared to experimental data. For LiK, deviation profiles have been simulated in order to prepare future experiments on this molecule.
\end{abstract}

PACS. 33.15.Kr Electric and magnetic moments (and derivatives), polarizability, and magnetic susceptibility - 33.55.Be Zeeman and Stark effects

\section{Introduction}

The deviation of molecular beams in a strong inhomogeneous electric field has been used to measure the electric polarizability of small molecules and clusters [1]. This is quite straightforward for non polar molecules. The deviation is due to the interaction of the electric field with the induced dipole of the molecule. In first approximation, one can interpret the experimental results by assuming that all the non polar molecules are deviated by the same amount which is proportional to the average polarizability of the molecule [1-3]. In contrary, for molecules with a permanent electric dipole, the deviation is due to the interaction of the electric field with both the induced dipole and the permanent dipole. This second term depends on the cosine of the angle $\theta$ between the molecular axis and the direction of the electric field. It induces a broadening of the molecular beam. This broadening has been observed in "two-wire" electric field experiments [4-6] or multipole electric field experiments [7-9]. The analysis of the deviation has to take into account the rotational motion of the molecule. The simplest approach is to consider the interaction energy with the external electric field as a perturbation to the rotational part of the Hamiltonian and to determine the first order and second order corrections to the unperturbed rotational levels of the molecule $[2,5,10]$.

\footnotetext{
a e-mail: benichou@lasim.univ-lyon1.fr

b UMR 5579 du CNRS
}

However this approach can only be applied if the interaction energy with the external field is small as compared to the rotational energy of the molecule. If not, the field induces a significant torque on the molecule and the rotational wave functions can be strongly modified. This effect has been used to produce molecular beams with aligned molecules in strong static field [11-16] or intense laser field [17]. Theoretically, the energy levels and the alignment of linear molecules (pendular states) have been studied by Friedrich and Herschbach [16,18]. They gave non perturbative solutions for polar-non polarizable molecules $(\mu \neq 0$ and $\alpha=0)$ and for non polar-polarizable molecules $(\mu=0, \alpha \neq 0)$. For the molecule LiNa that we have recently studied in electric deviation experiments [5] and for the electric field that we are using, the contributions of the induced dipole and of the permanent dipole are in the same order of magnitude and neither term can be neglected. In fact, for low $J$ values, the deviation is mainly due to the interaction with the permanent dipole, while the polarizability term is dominant for large $J$ values.

In order to interpret and to predict results for experiments which involved deviation of molecules in a static electric field, we have solved the problem of a rigid rotator in interaction with a strong electric field. This calculation is done for polar and polarizable linear molecules $(\mu \neq 0$ and $\alpha \neq 0$ ). The known problems of polar-non polarizable $(\mu \neq 0$ and $\alpha=0)$ and non polar-polarizable $(\mu=0$, $\alpha \neq 0$ ) molecules are particular cases of the present general process. Eigenenergies and eigenfunctions as well as 
derivatives of the energy with respect to the field are obtained. The values for the energy are also compared to approximate values obtained by a perturbative method in the low field limit and by an asymptotic expansion in the strong field limit. We focus our numerical results on heteronuclear alkali dimers such as LiNa for which we have obtained experimental results [5] and LiK and LiRb which, being by far more polar than LiNa, cannot be investigated accurately at our experimental field strength by perturbative approaches. The non perturbative approach is presented in Section 2. It is followed, in Section 3, by a discussion of eigenenergies, orientation parameters and molecular beam deviations of $\mathrm{LiNa}, \mathrm{LiK}$, and LiRb. Closing remarks are given in Section 4.

\section{Theory}

\subsection{Eigensolutions}

We consider a linear rotor in a ${ }^{1} \Sigma$ state interacting with a uniform electric field $\varepsilon$. The molecule has a permanent electric dipole moment $\mu$ along the molecular axis and a static dipole polarizability with two components $\alpha_{\|}$and $\alpha_{\perp}$ parallel and perpendicular to the molecular axis. The Schrödinger equation is:

$$
\begin{aligned}
H \psi & =E \psi \\
\text { with } \quad H & =B \mathbf{J}^{2}+V_{\alpha}(\theta)+V_{\mu}(\theta) .
\end{aligned}
$$

The first term in the right hand of equation (2) is the free rotational Hamiltonian of the molecule. The second and third terms are the potential parts of the Hamiltonian due to the induced and permanent dipole moment in the external field $\varepsilon$ :

$$
\begin{aligned}
& V_{\mu}(\theta)=-\mu \varepsilon \cos \theta, \\
& V_{\alpha}(\theta)=-\frac{\varepsilon^{2}}{2}\left(\left(\alpha_{\|}-\alpha_{\perp}\right) \cos ^{2} \theta+\alpha_{\perp}\right) .
\end{aligned}
$$

In equations $(2-4) \mathbf{J}$ is the angular momentum vector, $B$ the rotational constant, $\theta$ the polar angle between the molecular axis and the electric field direction. $E$ and $\Psi$ are the eigenenergy and eigenfunction to be determined.

The expression for $\mathbf{J}^{2}$ in spherical coordinates is:

$$
\mathbf{J}^{2}=-\left[\frac{1}{\sin \theta} \frac{\partial}{\partial \theta}\left(\sin \theta \frac{\partial}{\partial \theta}\right)+\frac{1}{\sin ^{2} \theta} \frac{\partial^{2}}{\partial \varphi^{2}}\right]
$$

$\varphi$ is the azimuthal angle. Equation (1) is separable and the wavefunction may be factorized:

$$
\psi(\theta, \varphi)=\mathrm{e}^{ \pm \mathrm{i} M \varphi} \phi(\theta)
$$

where $M$ is a good quantum number.

The Schrödinger equation obtained from equation (2) reduces to a spheroidal wave equation:

$$
\left[\frac{\mathrm{d}}{\mathrm{d} z}\left(1-z^{2}\right) \frac{\mathrm{d}}{\mathrm{d} z}-\frac{M^{2}}{1-z^{2}}+\Delta \omega z^{2}+\omega z+\lambda\right] \phi(\theta)=0
$$

where $z=\cos \theta$.

The dimensionless parameters $\Delta \omega$ and $\omega$ are given by:

$$
\begin{aligned}
\Delta \omega & =\frac{\left(\alpha_{\|}-\alpha_{\perp}\right) \varepsilon^{2}}{2 B} \text { (positive for linear systems) } \\
\omega & =\frac{\mu \varepsilon}{B} .
\end{aligned}
$$

The energy is given by:

$$
\lambda=\omega_{\perp}+\frac{E}{B} \quad \text { with } \quad \omega_{\perp}=\frac{\alpha_{\perp} \varepsilon^{2}}{2 B} .
$$

For $\Delta \omega=0$ and $\omega=0$, the eigenfunctions coincide with the spherical harmonics $Y_{J}^{M}$ and the eigenvalues are $E_{J, M} / B=J(J+1)-\omega_{\perp}$.

If the terms of interaction with the electric field are small compared to the rotational term, the eigenenergies and the eigenfunctions can be estimated with a perturbative approach $[2,5,10,19]$. In the low electric field limit, the perturbative energy for a level $J, M$ is given by:

$$
\begin{aligned}
\left(\frac{E}{B}\right)_{\text {pert. }} & =J(J+1)-\omega_{\perp}-\frac{\Delta \omega}{3} \\
+ & {\left[\frac{\omega^{2}}{2 J(J+1)}-2 \frac{\Delta \omega}{3}\right]\left[\frac{J(J+1)-3 M^{2}}{(2 J+3)(2 J-1)}\right] . }
\end{aligned}
$$

On the other hand, if the rotational energy is small as compared to the terms due to the interaction with the electric field, the rotation is blocked. In the high field limit, the energy of the system tends toward the energy of a twodimensional harmonic oscillator. The formula for $(\mu \neq$ $0, \alpha=0)$ and $(\mu=0, \alpha \neq 0)$ are given by Friedrich and Herschbach in reference [19]. Here, for $(\mu \neq 0, \alpha \neq$ 0 ) using the asymptotic expansion method described in reference [20] we obtain:

$$
\begin{aligned}
\left(\frac{E}{B}\right)_{\text {osc. }} & =-\omega-\Delta \omega-\omega_{\perp}+(2 \tilde{J}-M+1) \sqrt{2 \omega+4 \Delta \omega} \\
& -\frac{\Delta \omega}{2 \Delta \omega+\omega}\left[(2 \tilde{J}+1-M)^{2}+1-M^{2}\right] \\
-\frac{1}{8} & \frac{\omega}{2 \Delta \omega+\omega}\left[(2 \tilde{J}+1-M)^{2}+3-3 M^{2}\right]+\ldots \quad(12)
\end{aligned}
$$

In the general case, the Schrödinger equation can be solved to any accuracy by using a finite expansion of the wave functions in terms of spherical harmonics:

$$
\psi_{\tilde{J}, M}(\theta, \varphi)=\sum_{J=M}^{J_{\max }} a_{J}^{\tilde{J}, M} Y_{J}^{M}(\theta, \varphi) .
$$

For $\varepsilon \neq 0$ and for a linear system with $\mu$ and/or $\alpha$ non zero, $J$ is no longer a good quantum number while $M$ remains a good quantum number. The eigenstates are then labeled by $M$ and an integer $\tilde{J}$. $\tilde{J}$ is equal to the angular momentum $J$ of the eigenfunction without electric field that is adiabatically correlated to the function $\psi_{\tilde{J}, M}$. 
The expansion of the wave function in terms of spherical harmonics leads to the following symmetrical pentadiagonal matrix equation:

$$
[\mathcal{A}(\Delta \omega, \omega)]|a|=-\lambda|a| .
$$

The non vanishing elements are recalled in Appendix A. Eigenvalues $\lambda$ as well as expansion coefficients $a_{J}^{\tilde{J}, M}$ for the eigenfunctions are obtained by diagonalizing the matrix $[\mathcal{A}(\Delta \omega, \omega)]$. The eigenvalues $\lambda$ and the coefficients $a_{J}^{\tilde{J}, M}$ are functions of $\omega$ and $\Delta \omega$. In this paper, we have used the spheroidal wave equation (Eq. (7)) to solve this problem in order to follow the technique and the notations used by previous authors $[16,18]$. The pentadiagonal matrix (Eq. (14)) can also be obtained by projecting the Hamiltonian (Eqs. $(1,2))$ on the spherical harmonics basis and directly introducing the notation of the " $3 j$ " coefficients.

It should be noted that for polar-non polarizable molecules $(\Delta \omega=0)$ as well as for non polar-polarizable molecules $(\omega=0)$, the matrix $\mathcal{A}$ reduces to a tridiagonal form. For the latter case, $\boldsymbol{A}_{J, J}$ and $\boldsymbol{A}_{J, J \pm 2}$ terms are the only non zero elements of the matrix. The present process contains the two previously solved problems $(\mu \neq 0, \alpha=$ $0)$ and $(\mu=0, \alpha \neq 0)$ as particular cases.

\subsection{Alignment and orientation}

For a given eigenstate described by the wavefunction $\psi_{\tilde{J}, M}(\theta, \varphi)$, the probability distribution of $\theta$ is given by:

$$
n_{\tilde{J}, M}(\theta)=\int_{0}^{2 \pi} \psi_{\tilde{J}, M}^{*}(\theta, \varphi) \psi_{\tilde{J}, M}(\theta, \varphi) \mathrm{d} \varphi
$$

A second method to calculate this distribution is to expand $n(\theta)$ in terms of Legendre polynomials:

$$
n_{\tilde{J}, M}(\theta)=\sum_{n=0}^{\infty} b_{n}(\tilde{J}, M) P_{n}(\cos \theta)
$$

where

$$
b_{n}(\tilde{J}, M)=\frac{2 n+1}{2}\left\langle P_{n}(\cos \theta)\right\rangle_{\tilde{J}, M} .
$$

The expansion coefficients $b_{n}$ are functions of the expectation values of $\cos ^{n} \theta$ over the wavefunction $\psi_{\tilde{J}, M}(\theta, \varphi)$. Analytic expressions for $b_{n}(n=1 \ldots 4)$ are given in Appendix B. The values of $\langle\cos (\theta)\rangle$ and $\left\langle\cos ^{2}(\theta)\right\rangle$ can be interpreted as orientation and alignment parameters respectively $[16,18]$.

\subsection{Derivatives of eigenenergies with respect to the electric field}

In our experiments, static dipolar polarizability and/or permanent dipole moment are obtained by measuring the deflection of a cluster beam in an inhomogeneous electric field. In the electric field the force $g$ acting on the molecule is equal to the opposite of the gradient of the energy. This force is proportional to the gradient of the electric field and the first derivative of the energy with respect to the electric field:

$$
g=-\nabla E=-\frac{\partial E}{\partial \varepsilon} \frac{\partial \varepsilon}{\partial z}
$$

The evaluation of the first derivative of the energy with respect to the electric field $\partial E / \partial \varepsilon$ is necessary to interpret our experiments. This derivative is obtained by using the Hellman-Feynman theorem. The Hamiltonian $H$ (Eq. (2)) being hermitian and the wavefunctions $\psi_{\tilde{J}, M}$ being orthonormalized, the derivative $\partial E_{\tilde{J}, M} / \partial \varepsilon$ is given by:

$$
\begin{aligned}
\frac{\partial E_{\tilde{J}, M}(\varepsilon)}{\partial \varepsilon} & =\left\langle\psi_{\tilde{J}, M}\left|\frac{\partial H}{\partial \varepsilon}\right| \psi_{\tilde{J}, M}\right\rangle \\
-\frac{\partial E_{\tilde{J}, M}(\varepsilon)}{\partial \varepsilon} & =\left(\alpha_{\|}-\alpha_{\perp}\right) \varepsilon\left\langle z^{2}\right\rangle_{\tilde{J}, M}+\mu\langle z\rangle_{\tilde{J}, M}+\alpha_{\perp} \varepsilon .
\end{aligned}
$$

The force due to the permanent dipole is proportional to the orientation parameter $\langle z\rangle$ and the force due to the asymmetric part of the polarizability is proportional to the alignment parameter $\left\langle z^{2}\right\rangle$. The matrix elements $\langle z\rangle_{\tilde{J}, M}=\langle\cos \theta\rangle_{\tilde{J}, M}$ and $\left\langle z^{2}\right\rangle_{\tilde{J}, M}=\left\langle\cos ^{2} \theta\right\rangle_{\tilde{J}, M}$ are given in Appendix B (Eqs. (B.1, B.2)).

\section{Results}

Calculations have been performed for the three molecules $\mathrm{LiNa}, \mathrm{LiK}$ and LiRb in their ground state $\mathrm{X}^{1} \Sigma^{+}$. They require input data such as the permanent dipole $\mu$, the components of the static dipole polarizability $\alpha_{\|}$and $\alpha_{\perp}$ and the rotational constant $B . B$ is evaluated from the equilibrium internuclear distance $R_{\mathrm{e}}$ and the reduced molecular mass $m_{\mathrm{r}}$ :

$$
B=\frac{1}{2 m_{\mathrm{r}} R_{\mathrm{e}}^{2}} .
$$

For the ground state of LiNa, we use our experimental values of $\mu$ and $\alpha$, previously deduced from the measurement of the deviation of a LiNa supersonic beam in an inhomogeneous electric field [5]. In this previous study, we approximated the LiNa energy in the electric field by perturbation and used the experimental value of reference [21] for $R_{\mathrm{e}}$. For the ground state of $\mathrm{LiK}$ and LiRb, we have calculated $R_{\mathrm{e}}, \mu$ and the components $\alpha_{\|}$and $\alpha_{\perp}$ of the static dipole polarizability in a Density Functional Theory approach using Gaussian 94 [22]. We used the Perdew-Wang 91 [23] functional (DFT/PW91) and the Sadlej-Urban basis sets [24]. Values for $R_{\mathrm{e}}, B, \mu, \alpha_{\|}$and $\alpha_{\perp}$ are reported in Table 1. For LiNa, calculated values as well as experimental values are given for comparison. Calculated values for $\mu$ and $R_{\mathrm{e}}$ are in good agreement with experimental values with a relative error $\delta \approx 2 \%$. For the averaged polarizability $\bar{\alpha}=\left(\alpha_{\|}+2 \alpha_{\perp}\right) / 3, \delta \approx 9 \%$. The values of the dimensionless reduced parameters $\Delta \omega$ 
Table 1. Equilibrium distance, rotational constant, dipole moment and static dipole polarizability for LiNa, LiK and LiRb molecules.

\begin{tabular}{lccccccc}
\hline Molecule & & $R_{\mathrm{e}}(\AA)$ & $B\left(\mathrm{~cm}^{-1}\right)$ & $\mu(\mathrm{D})$ & $\alpha_{\|}\left(\AA^{3}\right)$ & $\alpha_{\perp}\left(\AA^{3}\right)$ & $\bar{\alpha}\left(\AA^{3}\right)$ \\
\hline $\mathrm{LiNa}$ & (a) & 2.89 & 0.379 & 0.49 & 52.3 & 32.3 & 39.0 \\
& (b) & 2.95 & 0.363 & 0.48 & 49.0 & 28.6 & 35.4 \\
$\mathrm{LiK}$ & (b) & 3.39 & 0.249 & 3.27 & 68.5 & 38.7 & 48.6 \\
$\mathrm{LiRb}$ & (b) & 3.51 & 0.213 & 4.40 & 75.6 & 41.6 & 52.9 \\
\hline
\end{tabular}

(a) Experimental values of reference [5].

(b) Present calculated values (DFT/PW91, SU Basis).

Table 2. Values of the dimensionless parameters $\Delta \omega, \omega$ and $\omega_{\perp}$ for an electric field $\varepsilon=2 \times 10^{7} \mathrm{~V} / \mathrm{m}^{-1}$ for $\mathrm{LiNa}$, LiK and LiRb.

\begin{tabular}{llcc}
\hline & $\Delta \omega=\frac{\left(\alpha_{\|}-\alpha_{\perp}\right) \varepsilon^{2}}{2 B}$ & $\omega=\frac{\mu \varepsilon}{B}$ & $\omega_{\perp}=\frac{\alpha_{\perp} \varepsilon^{2}}{2 B}$ \\
\hline LiNa & 0.059 & 4.34 & 0.095 \\
LiK & 0.134 & 45.74 & 0.174 \\
LiRb & 0.178 & 69.32 & 0.218 \\
\hline
\end{tabular}

(Eq. (8)), $\omega$ (Eq. (9)) and $\omega_{\perp}$ (Eq. (10)) are reported in Table 2 for an electric field $\varepsilon=2 \times 10^{7} \mathrm{Vm}^{-1}$ for the three molecules. While the two parameters of the induced dipole moment increase by a factor of 2 for $\omega_{\perp}$ and 3 for $\Delta \omega$, the parameter $\omega$ due to the permanent dipole increases by a factor of 16 from LiNa to LiRb.

Table 3 compares the rotational energy of the free rotor to the second order corrections in energy due to the induced and the permanent dipoles. The corrections are calculated using equation (11). This table shows that the correction due to the polarizability little depends on the rotational state of the molecule while the perturbative correction due to the permanent dipole decreases as $J$ increases. For a ratio of the potential terms with respect to the rotational energy small compared to 1 , perturbation theory is an appropriate approach. For larger ratios perturbation theory fails and the non perturbative approach is the appropriate method.

\subsection{Eigenenergies}

For each molecule and for the various values of the electric field $\varepsilon$, eigenenergies and eigenfunctions are obtained by diagonalizing the matrix $\mathcal{A}$ for each value of $M$ (Eq. (14)). The non vanishing elements of this matrix are evaluated for the molecular values of $\mu, \alpha_{\|}, \alpha_{\perp}$ and $B$ reported in Table 1 with the expressions given in Appendix A.

The number of terms in the expansion of the wave function (Eq. (13)) is determined so that eigenenergy values are stabilized to a given accuracy when increasing $J_{\max }$. In the present calculations, we used

$$
\left|\left(\frac{E}{B}\right)_{J_{\max }+1}-\left(\frac{E}{B}\right)_{J_{\max }}\right| \leq 10^{2}
$$

Table 3. Rotational energy of the free rotor and perturbative corrections to this energy due the potential terms $V_{\mu}$ and $V_{\alpha}$. The corrections are evaluated for $\varepsilon=2 \times 10^{7} \mathrm{Vm}^{-1}$ using (Eq. (11)). These corrections are labeled $E_{\mu}$ and $E_{\alpha}$. Results are given for selected rotational levels.

\begin{tabular}{cccccc}
\hline & $\mathrm{J}$ & $\mathrm{M}$ & $\frac{J(J+1)}{B}$ & $\frac{E_{\mu}}{B}$ & $\frac{E_{\alpha}}{B}$ \\
\hline $\mathrm{LiNa}$ & 0 & 0 & 0 & -3.15 & -0.115 \\
& 5 & 0 & 30 & 0.081 & -0.13 \\
& 5 & 5 & 30 & -0.12 & -0.10 \\
& 10 & 0 & 110 & 0.021 & -0.13 \\
& 10 & 10 & 110 & -0.037 & -0.098 \\
\hline $\mathrm{LiK}$ & 0 & 0 & 0 & -348.70 & -0.22 \\
& 5 & 0 & 30 & 8.94 & -0.24 \\
& 5 & 5 & 30 & -13.41 & -0.18 \\
& 10 & 0 & 110 & 2.39 & -0.24 \\
& 10 & 10 & 110 & -4.13 & -0.18 \\
\hline LiRb & 0 & 0 & 0 & -800.77 & -0.28 \\
& 5 & 0 & 30 & 20.53 & -0.31 \\
& 5 & 5 & 30 & -30.80 & -0.23 \\
& 10 & 0 & 110 & 5.50 & -0.31 \\
& 10 & 10 & 110 & -9.50 & -0.23 \\
\hline
\end{tabular}

which corresponds for $J=15, M=0$ and $\varepsilon=3 \times$ $10^{8} \mathrm{Vm}^{-1}$ to $J_{\max }=22$ for LiNa, $J_{\max }=38$ for $\mathrm{LiK}$ and $J_{\max }=43$ for LiRb.

Eigenenergies $E / B$ for $\varepsilon=2 \times 10^{7} \mathrm{Vm}^{-1}$ are given in Table 4 for the states $\tilde{J}=0-3,5,10$ with $M=0 \ldots \tilde{J}$ for the three molecules LiNa, LiK, LiRb. The energies for the states $\tilde{J}=0,5,15$ are plotted as a function of $\varepsilon$ in Figures 1 and 2 for LiNa and LiK respectively. The corresponding curves for LiRb are similar to that for LiK and are not reported here. The main effect of the electric field is to break the degeneracy in $M$. The energy of a molecule depends on its orientation in the electric field. For $\mathrm{LiNa}$ and $\tilde{J}=15$, the contribution to the energy due to the permanent dipole and the asymmetric part of the polarizability are small as compared to the rotational energy of the molecule. Moreover, for $\tilde{J} \approx 15$ they cancel each other. In this case, the only effect of the electric field is a small decrease in the energy of the molecule. For different values of $J$, there would be a small splitting of the 
Table 4. Exact eigenenergies $E / B$ and perturbative values (Eq. (11)) for given $\tilde{J}$ and $M$ states of LiNa, LiK and LiRb at $\varepsilon=2 \times 10^{7} \mathrm{Vm}^{-1}$. Values calculated with the formula for high field approximation (Eq. (12)) are given for LiRb.

\begin{tabular}{|c|c|c|c|c|c|c|c|c|}
\hline & & \multicolumn{3}{|c|}{$\mathrm{LiNa}$} & & \multicolumn{2}{|l|}{$\mathrm{LiRb}$} \\
\hline$\tilde{J}$ & $M$ & $\begin{array}{c}\text { Exact } \\
\text { solution }\end{array}$ & $\begin{array}{c}\text { Perturbative } \\
\text { energy }\end{array}$ & $\begin{array}{c}\text { Exact } \\
\text { solution }\end{array}$ & $\begin{array}{c}\text { Perturbative } \\
\text { energy }\end{array}$ & $\begin{array}{c}\text { Exact } \\
\text { solution }\end{array}$ & $\begin{array}{c}\text { Perturbative } \\
\text { energy }\end{array}$ & $\begin{array}{c}\text { High field } \\
\text { approximation }\end{array}$ \\
\hline 0 & 0 & -2.06 & -3.26 & -36.97 & -348.7 & -58.42 & -801.0 & -58.59 \\
\hline \multirow[t]{2}{*}{1} & 0 & 2.51 & 3.76 & -18.86 & 210.97 & -35.87 & 482.14 & -36.00 \\
\hline & 1 & 1.04 & 0.95 & -27.36 & -102.81 & -46.60 & -238.49 & -46.79 \\
\hline \multirow[t]{3}{*}{2} & 0 & 6.36 & 6.32 & -1.93 & 55.57 & -14.46 & 120.08 & -14.42 \\
\hline & 1 & 6.02 & 6.10 & -9.78 & 30.68 & -24.58 & 62.90 & -24.70 \\
\hline & 2 & 5.46 & 5.45 & -17.13 & -44.01 & -34.20 & -108.64 & -34.49 \\
\hline \multirow[t]{4}{*}{3} & 0 & 12.09 & 12.08 & 13.67 & 35.00 & 5.71 & 65.08 & 6.15 \\
\hline & 1 & 12.03 & 12.03 & 6.60 & 29.20 & -3.72 & 51.74 & -3.63 \\
\hline & 2 & 11.87 & 11.88 & 0.04 & 11.78 & -12.62 & 11.72 & -12.91 \\
\hline & 3 & 11.64 & 11.64 & -6.15 & -17.25 & -21.10 & -54.97 & -21.70 \\
\hline \multirow[t]{6}{*}{5} & 0 & 29.96 & 29.96 & 39.38 & 38.70 & 41.60 & 50.22 & 44.23 \\
\hline & 1 & 29.954 & 29.95 & 35.06 & 37.81 & 33.98 & 48.17 & 35.46 \\
\hline & 2 & 29.93 & 29.93 & 30.90 & 35.13 & 26.96 & 42.02 & 27.20 \\
\hline & 3 & 29.89 & 29.89 & 26.81 & 30.67 & 20.30 & 31.77 & 19.43 \\
\hline & 4 & 29.84 & 29.84 & 22.76 & 24.43 & 13.88 & 17.42 & 12.16 \\
\hline & 5 & 29.78 & 29.78 & 18.75 & 16.40 & 7.65 & -1.03 & 5.38 \\
\hline \multirow[t]{11}{*}{10} & 0 & 109.90 & 109.90 & 112.19 & 112.15 & 115.39 & 115.19 & 121.65 \\
\hline & 1 & 109.90 & 109.90 & 112.11 & 112.09 & 115.19 & 115.04 & 115.43 \\
\hline & 2 & 109.90 & 109.90 & 111.90 & 111.89 & 114.59 & 114.59 & 109.70 \\
\hline & 3 & 109.90 & 109.89 & 111.54 & 111.57 & 113.64 & 113.85 & 104.47 \\
\hline & 4 & 109.90 & 109.89 & 111.05 & 111.12 & 112.39 & 112.80 & 99.74 \\
\hline & 5 & 109.89 & 109.89 & 110.43 & 110.54 & 110.88 & 111.46 & 95.50 \\
\hline & 6 & 109.89 & 109.89 & 109.70 & 109.82 & 109.17 & 109.82 & 91.77 \\
\hline & 7 & 109.88 & 109.88 & 108.86 & 108.98 & 107.28 & 107.88 & 88.53 \\
\hline & 8 & 109.88 & 109.88 & 107.92 & 108.01 & 105.24 & 105.65 & 85.78 \\
\hline & 9 & 109.87 & 109.87 & 106.90 & 106.91 & 103.08 & 103.11 & 83.54 \\
\hline & 10 & 109.87 & 109.87 & 105.80 & 105.69 & 100.82 & 100.28 & 81.79 \\
\hline
\end{tabular}

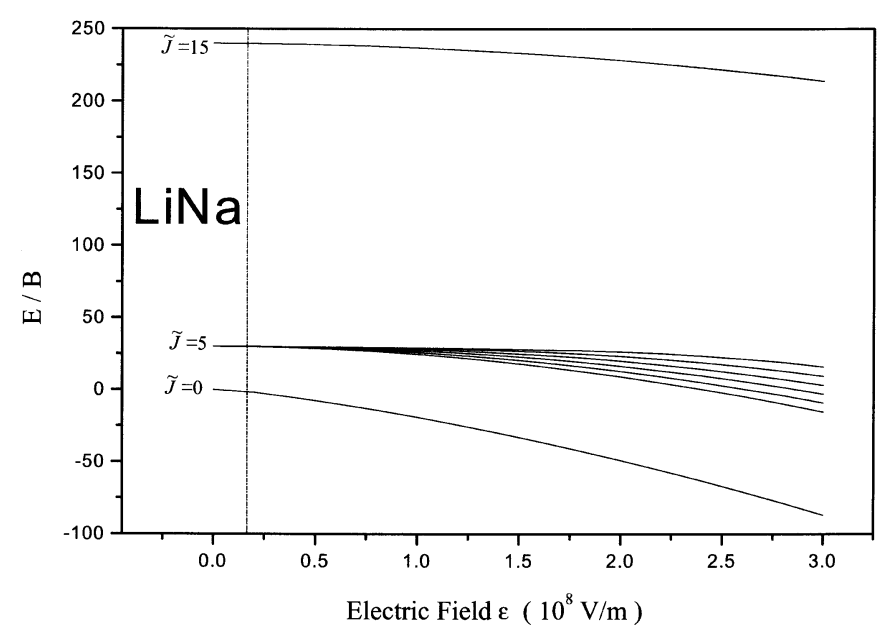

Fig. 1. Energy $E / B$ calculated with the non perturbative approach for the states $\tilde{J}=0,5,15 ; M=0-\tilde{J}$ of LiNa plotted as a function of the electric field $\varepsilon$. The value of the experimental electric field is indicated by the vertical dotted line.

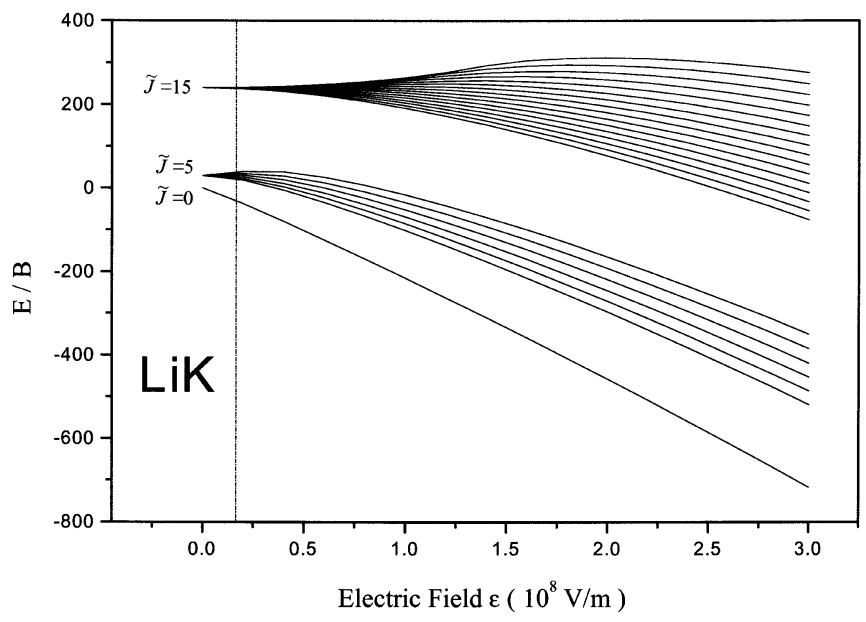

Fig. 2. Energy $E / B$ calculated with the non perturbative approach for the states $\tilde{J}=0,5,15 ; M=0-\tilde{J}$ of LiK plotted as a function of the electric field $\varepsilon$. The value of the experimental electric field is indicated by the vertical dotted line. 
rotational sublevels. The compensation of the two terms would be for a different value of $J$ for another molecule.

In Table 4, we also give the results of the perturbative approach (Eq. (11)). As expected, the perturbative approach fails when the potential terms are not small compared to the rotational energy of the free rotor (see Tab. 3). At $\varepsilon=2 \times 10^{7} \mathrm{Vm}^{-1}$ for LiNa, the perturbative approximation in the low field limit cannot be used for the states $\tilde{J}=0,1$. For $\tilde{J} \geq 3$, the perturbative approximation is excellent and its accuracy increases with increasing $\tilde{J}$ to reach $\delta \approx 10^{-4 \%}$ for $\tilde{J}=15$. It should be noted that approximating the energy $E / B$ with equation (11) as we did in our previous work to deduce $\mu$ and $\bar{\alpha}$ from our experiments on LiNa was totally justified. The situation is not the same for LiK and LiRb. These molecules have a stronger permanent dipole and the accuracy of the perturbative approximation is not good.

The results of the high field approximation (Eq. (12)) are reported here (Tab. 4) only for LiRb which has the strongest permanent dipole. For rotational levels with $\tilde{J} \leq$ 5 , this approximation is better than the value obtained from equation (11) and is quite good for the first rotational levels.

\subsection{Orientation parameters}

In our experiment, as mentioned is Section 2, the deviation of the molecules is related to the orientation and alignment parameters in the electric field. In other experiments like collision experiments with oriented molecules, the calculation of this parameter is also needed to interpret experimental results.

The expectation values of $\left\langle\cos ^{n} \theta\right\rangle$ for $n=1 \ldots 4$ have been evaluated with the formulas (B.1-B.4) for various values of $\tilde{J}$ and averaged over $M$ states:

$$
\left\langle\cos ^{n} \theta\right\rangle_{\tilde{J}}=\frac{1}{2 \tilde{J}+1} \sum_{M=-\tilde{J}}^{\tilde{J}}\left\langle\cos ^{n} \theta\right\rangle_{\tilde{J}, M} .
$$

As an illustrative example, the variation of $\langle\cos \theta\rangle_{\tilde{J}}$ with the electric field $\varepsilon$ is plotted in Figure 3 for the states $\tilde{J}=0-5$ of LiNa. The quantity $\langle\cos \theta\rangle_{\tilde{J}}$ is strongly related to the dipolar term $\omega=\mu \varepsilon / B$. It measures the degree of orientation of the molecule. Only polar molecules can be oriented in a static field (Eq. (B.1)). Non polar diatomic molecules cannot be oriented but they can be aligned $\left(\left\langle\cos ^{2} \theta\right\rangle_{\tilde{J}, M} \neq 0\right)[18]$. In Figure 3 , the largest values of $\langle\cos \theta\rangle_{\tilde{J}}$ are obtained for $\tilde{J}=0$. Orientation for $\tilde{J} \geq 5$ at the displayed range of field strength is negligible.

To study the molecular orientation, it is convenient to display the distribution $n(\theta)$ for a given electric field. The distribution of the molecular axis $n(\theta)$ (Eq. (15)) averaged over states $M$ has been calculated for $\varepsilon=2 \times 10^{7} \mathrm{Vm}^{-1}$ and $\tilde{J}=1,3,5$. Results for LiNa and LiK are displayed in Figures 4 and 5, respectively. As discussed above, for low values of $\tilde{J}$, the electric field induces an orientation of the molecule along the $z$-axis (electric field axis). For LiNa,

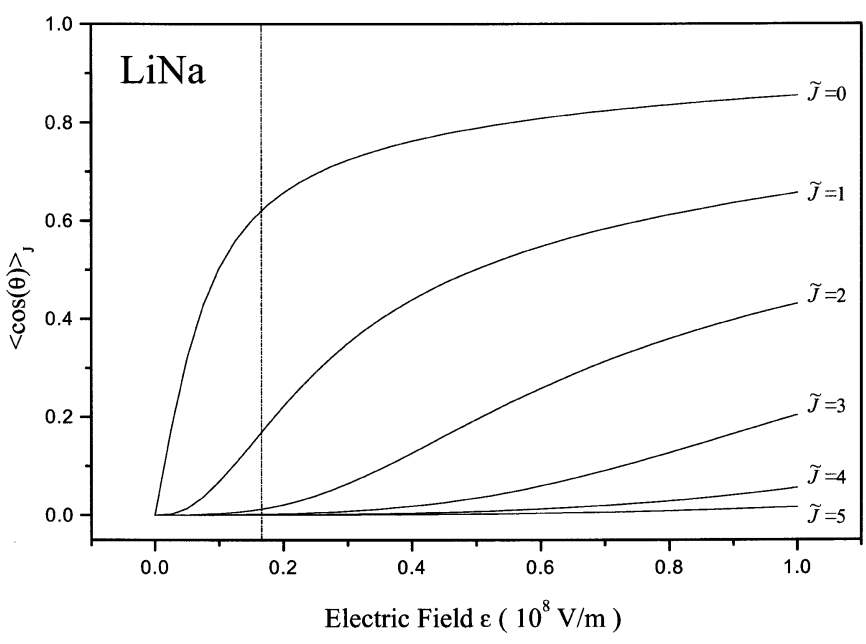

Fig. 3. Averaged value $\langle\cos \theta\rangle_{\tilde{J}}$ for some states of LiNa plotted as a function of the electric field $\varepsilon$. The value of the experimental electric field is indicated by the vertical dotted line.

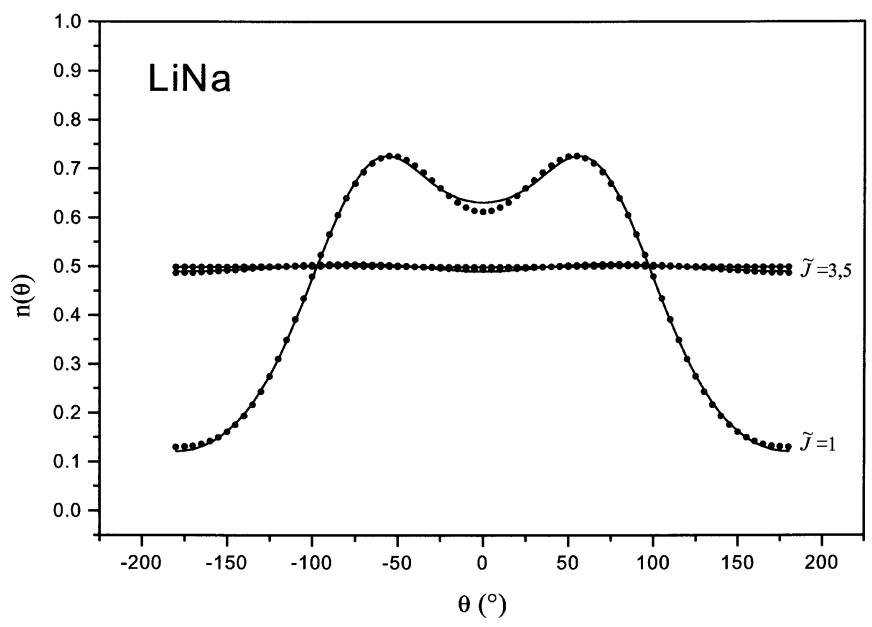

Fig. 4. Averaged angular distribution of the molecular axis for the states $\widetilde{J}=1,3,5$ of $\mathrm{LiNa}$ (calculations are done for $\varepsilon=$ $2 \times 10^{7} \mathrm{Vm}^{-1}$ ). Full lines correspond to the exact calculation (Eq. (15)) and dots to the values obtained from the Legendre expansion (Eq. (16), 4th order).

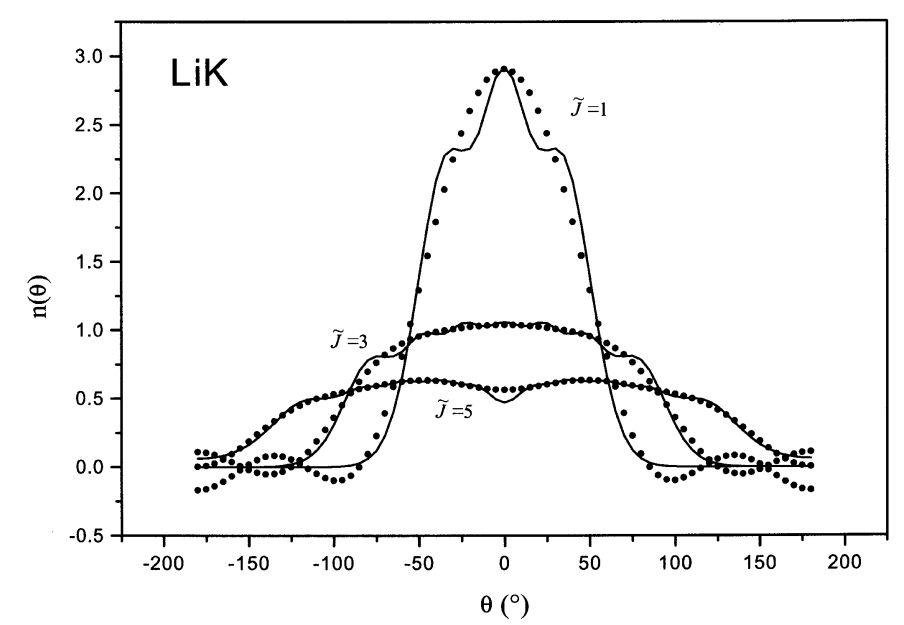

Fig. 5. Same as Figure 4 for the states $\tilde{J}=1,3,5$ of LiK. 


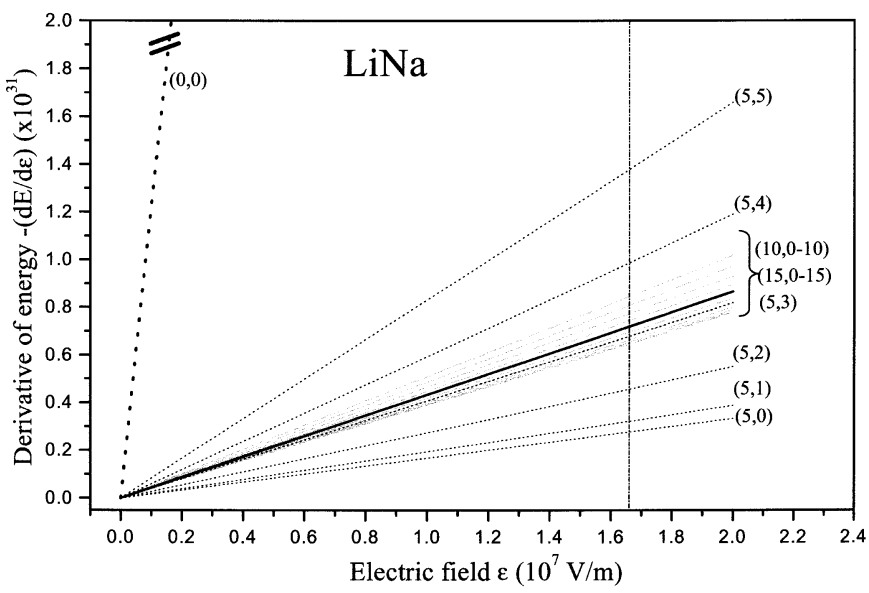

Fig. 6. Derivative of the energy with respect to the electric field (Eq. (20)) for the states $\tilde{J}=0,5,10,15$ of LiNa. The sublevels $M$ are indicated by the notation $(\tilde{J}, M)$. The value of the experimental electric field is indicated by the vertical dotted line.

orientation occurs for $\tilde{J}=1$, but for $\tilde{J}=3$ and $\tilde{J}=5$, the distribution is flat. For LiK, the orientation around $\theta=0$ occurs for $\tilde{J}=1,3,5$. For this molecule and for these states, the value of $\mu \varepsilon$ is greater than the rotational energy of the molecule $(\omega \gg 1)$. The rotation is hindered. In Figures 4 and 5, we have also plotted the distribution obtained from the expansion in Legendre polynomials (Eq. (16)). For both molecules, expansions to the fourth order are a good representation of the exact distributions for the value of the electric field considered here. Convergence of (Eq. (16)) was considered for the state $\tilde{J}=1$ of LiK. A reasonable description has to include terms up to $\left\langle\cos ^{3} \theta\right\rangle_{\tilde{J}}$.

\subsection{Derivatives of the energy}

The derivative of the energy with respect to the electric field corresponds to the quantity which is measured in beam deviation experiments. In an inhomogeneous electric field, the force on the molecule is proportional to this derivative (Eq. (18)). The energy gradient with respect to field strength $(-\partial E / \partial \varepsilon)$ for LiNa is reported in Figure 6 for selected states. The derivative depends strongly on the rotational level of the molecule. For $\tilde{J}=0$, the derivative increases rapidly with the value of the electric field. For rotational states of low $\tilde{J}$ values, the force due to the inhomogeneous electric field will induce a separation of the different $M$ sublevels. For states with $\tilde{J} \geq 10$, the value of the derivative is mainly due to the term proportional to the average polarizability. This term does not depend on the values of $\tilde{J}$ or $M$. For $\tilde{J} \geq 10$, the force will little depend on the rotational level of the molecule. Moreover, for all the states with $\tilde{J} \geq 2$, there is no orientation of the molecule in the electric field (see Figs. 3 and 4, Tab. 4), the perturbative approach is correct and the evolution of the value of $(-\partial E / \partial \varepsilon)$ is linear with the electric field.

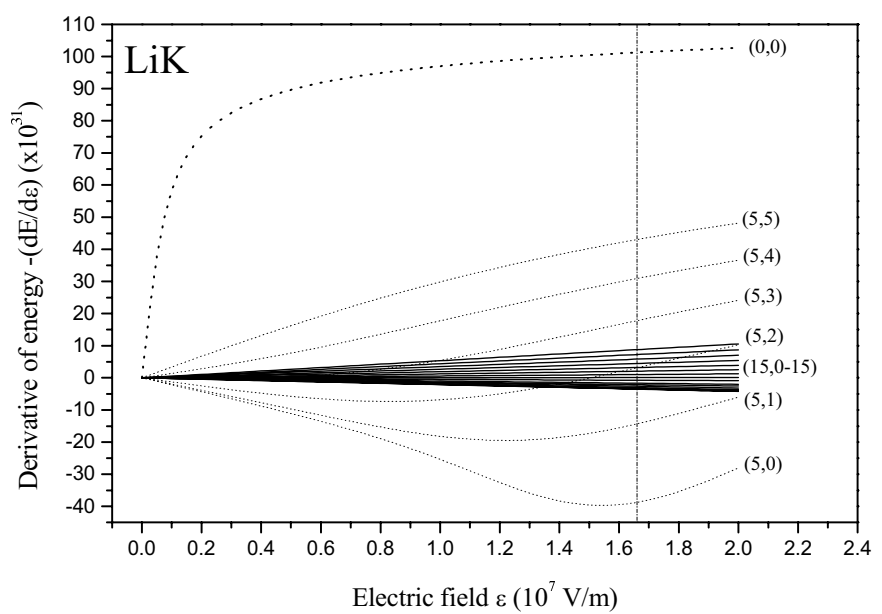

Fig. 7. Same as Figure 6 for LiK (vertical scales in Figs. 6 and 7 are different).

The derivative of the energy with respect to the electric field $(-\partial E / \partial \varepsilon)$ is plotted in Figure 7 for several states of the molecule LiK. For LiK, the force is mainly due to the interaction of the electric field with the permanent dipole. Several differences between Figures 6 and 7 are observed. First, note that the vertical scales in the two figures are different. The derivative $(-\partial E / \partial \varepsilon)$ and then the forces on this molecule are more than one order of magnitude larger than for the molecule LiNa. Second, for LiK the orientation of the molecules cannot be neglected. For $\tilde{J}=0$ and an electric field strength of the order of $10^{7} \mathrm{Vm}^{-1},(-\partial E / \partial \varepsilon)$ is almost constant. LiK molecules in $\tilde{J}=0$ state are strongly oriented. The derivative which is here in first approximation equal to $\mu\langle\cos \theta\rangle$ does not depend on the value of $\varepsilon$. For $\tilde{J}=5$, the derivative of the energy for several sublevels is negative. These sublevels are anti-oriented $\left(\langle\cos \theta\rangle_{\tilde{J}, M}<0\right)$ and the molecules will be deviated toward the low electric field region.

\subsection{Deflection of molecular beam}

In this section we simulate experimental data of a deflected molecular beam of LiNa [5] on the basis of the above outlined procedure. The experimental set up and results are described in reference [5]. Briefly, the LiNa molecules are produced in a seeded molecular beam. The molecular beam is collimated by two slits. It is deviated $1 \mathrm{~m}$ after the source in a $15 \mathrm{~cm}$ long deflector which produces a strong inhomogeneous electric field. The experimental value of the electric field on the beam axis is equal to $\varepsilon=1.7 \times 10^{7} \mathrm{Vm}^{-1}$. The molecules are excited and ionized (Two Photons Ionization technique) $1 \mathrm{~m}$ after the deflector in the extraction region of a time of flight mass spectrometer. The deviation $d$ is measured in the region of ionization (see Ref. [5] for details). The force (Eq. (18)) and the deviation $d$ of the beam are proportional to the gradient of the energy of the molecule in the electric field:

$$
d=-\frac{K}{2 m v^{2}} \nabla E=-\frac{K}{2 m v^{2}} \frac{\partial E}{\partial \varepsilon} \frac{\partial \varepsilon}{\partial z}
$$




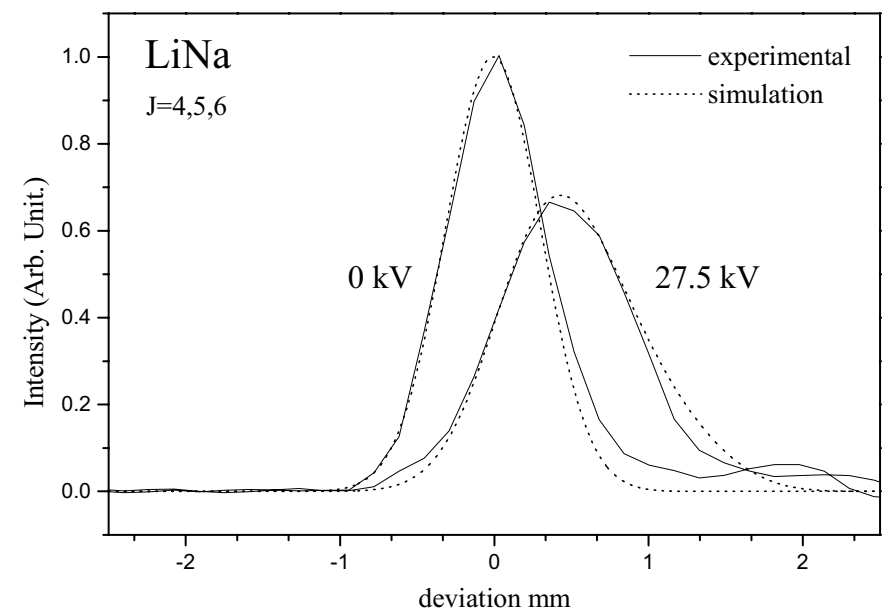

Fig. 8. Beam deflection profiles for $J=4,5,6$ levels of the molecule $\mathrm{NaLi}$ with and without electric field in the deviator: experimental data (full lines), simulation obtained from the derivative of the energy (dashed lines).

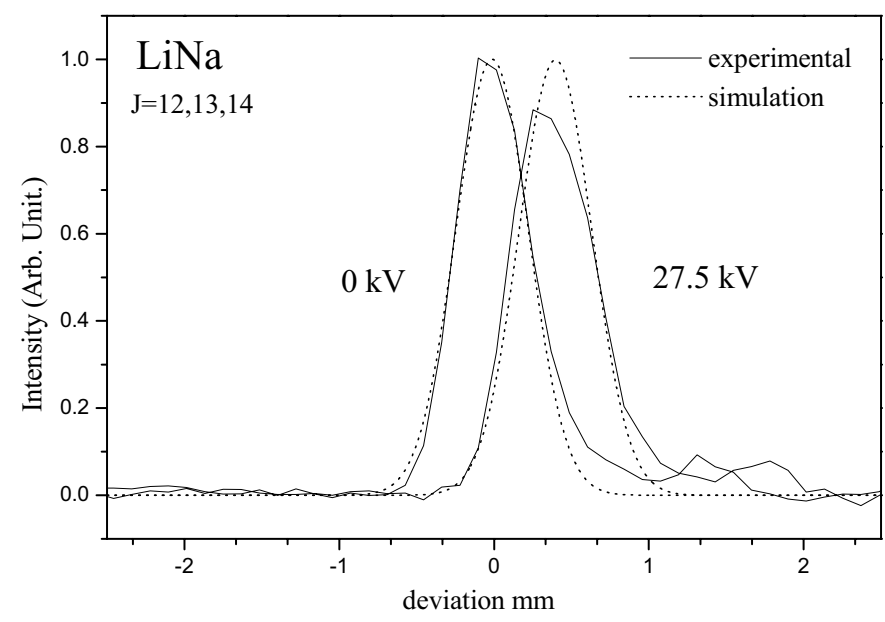

Fig. 9. Same as Figure 8 for $J=12,13,14$ levels.

$m$ and $v$ are the mass and the velocity of the molecules, $K$ is a geometrical factor and $z$ is the direction of the electric field. The molecules are excited and ionized with $\mathrm{XeCl}$ pumped dye lasers. The first photon is resonant on the $\mathrm{B}^{1} \Pi\left(v^{\prime}=7\right) \leftarrow \mathrm{X}^{1} \Sigma\left(v^{\prime \prime}=0\right)$ vibronic transition. This allows to select a single or a few rotational states of the molecule. Profiles of the molecular beam measured with and without electric field in the deflector for molecules with low rotational angular momentum $(J=4,5,6)$ and for molecules with high angular momentum $(J=12,13$, 14) are given in Figures 8 and 9. For low $J$ values, the electric field induces a shift and a broadening of the beam. For high $J$ values, the only effect of the electric field is a shift of the molecular beam.

Calculated deviations are obtained from the derivative of the eigenenergies and using (Eq. (22)). To compare calculated profiles to experimental profiles, a Gaussian profile in the $z$-direction is assumed for the shape of the beam before the deflection. Figures 8 and 9 show an excellent agreement between calculated and experimental profiles.

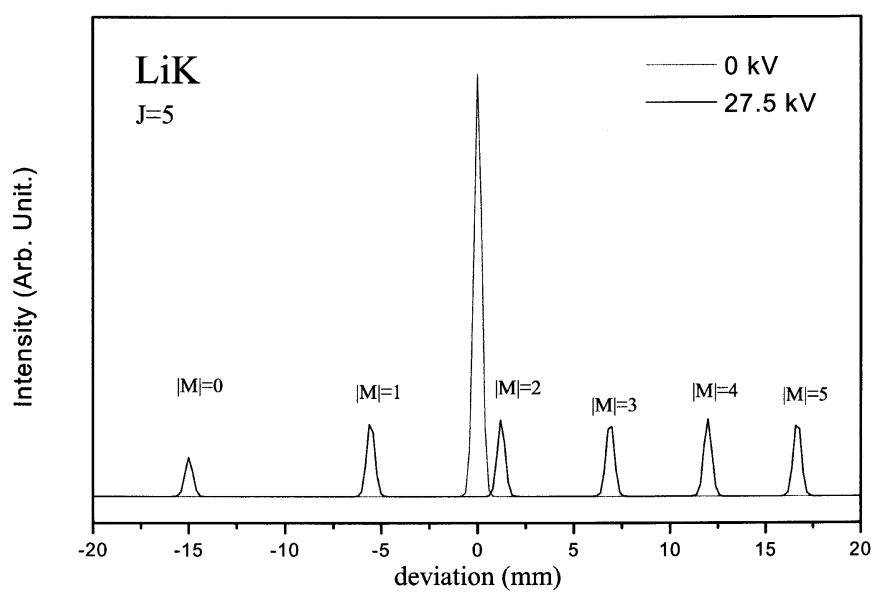

Fig. 10. Simulation of a beam deflection profile for $J=5$ levels of the molecule $\mathrm{LiK}$ with and without electric field in the deviator.

In particular, the broadening of the beam for low $J$ values is well reproduced. The broadening is due to the potential term $V_{\mu}$. For large values of $J$, the influence of this term decreases (see Tab. 3) and no significant broadening is expected (see Fig. 6).

Figure 10 shows the calculated deviations for the LiK molecule for $J=5$. As already mentioned, the effect of the electric field is much stronger for LiK than for LiNa. The term due to the permanent dipole is larger for LiK than for $\mathrm{LiNa}\left((\omega)_{\mathrm{LiK}} /(\omega)_{\mathrm{LiNa}} \approx 16\right)$. For low $J$ value, the electric field would induce a strong spreading of the beam with a separation of the different sublevels. Each peak in Figure 10 corresponds to a given Stark sublevel of the molecule (a well defined $|M|$ value). Due to the anti-orientation of some sublevels, the beam is spread in both directions in the deflector (toward high and low electric field regions). Beyond the determination of the values of the static polarizability and the permanent dipole, the profile of deviation gives a picture of the orientation of the molecule due to the electric field in the deviator. Moreover, as this has already been shown with four-wire alternate electrical fields [9], it is also possible to use such experimental set up to select a rotational state of the molecule. Figure 11 shows the deviation profile obtained by including the entire rotational distribution of the LiK molecules in the beam for a rotational temperature of $10 \mathrm{~K}$. For this temperature and this molecule, rotational states with $J<10$ are the only one with a significant population. The peaks corresponding to the low values of $J(J<6)$ are well separated in space. This figure shows that it is possible to use our experimental set up to select one given stark sublevel of a molecule and to perform experiments on the selected level. The rotational state selection can be applied to any molecule with dimensionless parameter $\omega$ (for $\varepsilon=1.7 \times 10^{7} \mathrm{Vm}^{-1}$ ) equal or larger to that of LiK. 


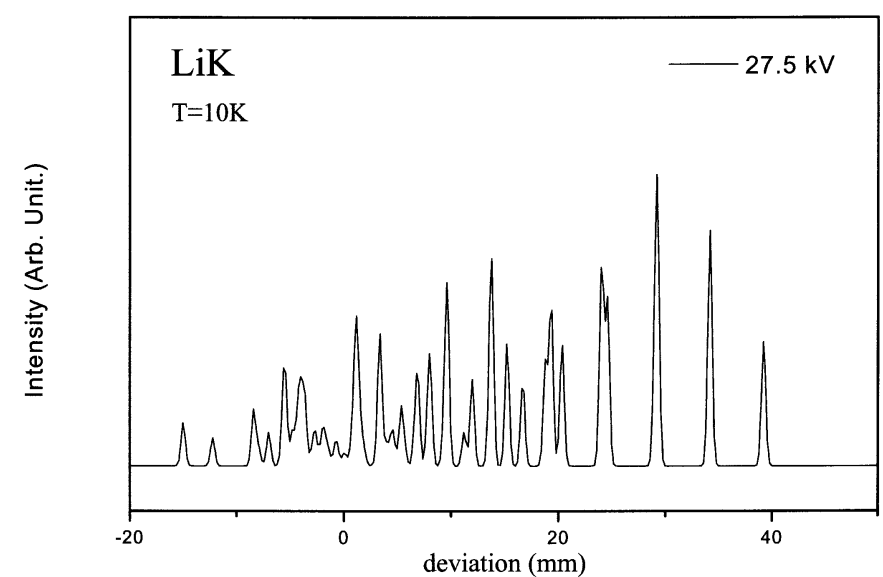

Fig. 11. Simulation of the profile of deviation obtained by including the entire rotational distribution of the LiK molecules in the beam for a rotational temperature of $10 \mathrm{~K}$. All the parameters used for this simulation correspond to our experimental conditions.

\section{Conclusion}

A non perturbative approach is used to solve the problem of a polar and polarizable linear molecule in an inhomogeneous electric field, in the assumption of a rigid rotator model. Eigenenergies and eigenfunctions are obtained by diagonalizing a symmetrical pentadiagonal matrix. The derivative of the eigenenergies with respect to the electric field is evaluated using the Hellman-Feynman theorem. This calculation gives a tool to interpret and predict beam deflection experiments of polar and polarizable molecules.

Results have been presented for the three molecules $\mathrm{LiNa}$, LiK and LiRb which, with respect to the problem solved here, differ mainly by the value of their permanent dipole. Simulations show that it is possible to use our experimental set up to work on selected rotational levels of the molecule.

\section{Appendix A: Elements of the symmetrical pentadiagonal matrix $[\mathcal{A}(\Delta \omega, \omega)]$}

The matrix elements of $[\boldsymbol{A}(\Delta \omega, \omega)]$ for a given value of $M$ are:

$$
\mathcal{A}_{J, J}(\Delta \omega, \omega)=-J(J+1)+\Delta \omega\left(\frac{2 J^{2}-2 M^{2}+2 J-1}{(2 J+3)(2 J-1)}\right)
$$

$$
\begin{aligned}
& \mathcal{A}_{J, J+1}(\Delta \omega, \omega)=\mathcal{A}_{J+1, J}(\Delta \omega, \omega) \\
&=\omega\left(\frac{(J+M+1)(J-M+1)}{(2 J+3)(2 J+1)}\right)^{1 / 2} \\
& \mathcal{A}_{J, J+2}(\Delta \omega, \omega)=\mathcal{A}_{J+2, J}(\Delta \omega, \omega)=\frac{\Delta \omega}{2 J+3} \\
& \times\left(\frac{(J+M+1)(J-M+1)(J+M+2)(J-M+2)}{(2 J+1)(2 J+5)}\right)^{1 / 2}
\end{aligned}
$$

$$
\mathcal{A}_{J, J+l}(\Delta \omega, \omega)=\mathcal{A}_{J+l, J}(\Delta \omega, \omega) \equiv 0 \quad \text { for } l \geq 3
$$

with $J \equiv M \ldots J_{\max }$ and $\Delta \omega=\frac{\left(\alpha_{\|}-\alpha_{\perp}\right) \varepsilon^{2}}{2 B}, \omega=\frac{\mu \varepsilon}{B}$.

\section{Appendix B: Average values $\left\langle\cos ^{n} \theta\right\rangle_{\tilde{J}, M}$ for $n=1 \ldots 4$}

$$
\begin{gathered}
\langle\cos \theta\rangle_{\tilde{J}, M}=2 \sum_{J=M}^{\infty} a_{J} a_{J+1} A(J) \\
\left\langle\cos ^{2} \theta\right\rangle_{\tilde{J}, M}=2 \sum_{J=M}^{\infty} a_{J} a_{J+2} A(J) A(J+1) \\
+\sum_{J=M}^{\infty} a_{J} a_{J}\left\{A^{2}(J)+A^{2}(J-1)\right\} \\
\left\langle\cos ^{3} \theta\right\rangle_{\tilde{J}, M}=2 \sum_{J=M}^{\infty} a_{J} a_{J+3} A(J) A(J+1) A(J+2) \\
+2 \sum_{J=M}^{\infty} a_{J} a_{J+1} A(J)\left\{A^{2}(J-1)+A^{2}(J)+A^{2}(J+1)\right\} \\
+\sum_{J=M}^{\infty} a_{J} a_{J}\left[A^{2}(J)\left\{A^{2}(J-1)+A^{2}(J)+A^{2}(J+1)\right\}\right. \\
+\sum_{J=M}^{\infty} a_{J} a_{J+2} A(J) A(J+1)\left\{A^{2}(J)+A^{2}(J+1)+A^{2}(J+2)\right\} \\
\left\langle\cos ^{4} \theta\right\rangle_{\tilde{J}, M}=2 \sum_{J=M}^{\infty} a_{J} a_{J+4} A(J) A(J+1) A(J+2) A(J+3) \\
\left.\left.+A^{2}(J-2)+A^{2}(J-1)+A^{2}(J)\right\}\right] .
\end{gathered}
$$

In formulas (B.1-B.4), the shortened notation $a_{J} \equiv a_{J}^{\tilde{J}, M}$ has been used.

The function $A(J)$ is defined by:

$$
\begin{aligned}
& A(J)=\left[\frac{(J+1-M)(J+1+M)}{(2 J+1)(2 J+3)}\right]^{1 / 2} \\
& \text { with } A(J<0)=0 \text {. }
\end{aligned}
$$

\section{References}

1. K.D. Bonin, V.V. Kresin, Electric-dipole polarizabilities of atoms, molecules and clusters (World Scientific, Singapore, 1997).

2. V. Tarnovsky, M. Bunimovicz, L. Vuskivic, B. Stumf, B. Bederson, J. Chem. Phys. 98, 3894 (1993).

3. T.M. Miller, B. Bederson, in Advances in Atomic and Molecular Physics (Academic Press, New York, 1977), Vol. 13, p. 1. 
4. T.L. Story, A.J. Hebert, J. Chem. Phys. 64, 855 (1976).

5. R. Antoine, D. Rayane, A.R. Allouche, M. Aubert-Frécon, E. Benichou, F.W. Dalby, P. Dugourd, M. Broyer, C. Guet, J. Chem. Phys. 110, 5568 (1999).

6. R. Schäfer, S. Schlecht, J. Woenckhaus, J.A. Becker, Phys. Rev. Lett. 76, 471 (1996).

7. P.R. Brooks, E.M. Jones, K. Smith, J. Chem. Phys. 51, 3073 (1969).

8. K.H. Kramer, R.B. Bernstein, J. Chem. Phys. 42, 767 (1965).

9. A. Lübbert, G. Rotzoll, F. Günther, J. Chem. Phys. 69, 5174 (1978).

10. M. Brieger, Chem. Phys. 89, 275 (1984).

11. A. Durand, J.C. Loison, J. Vigué, J. Chem. Phys. 106, 477 (1997).

12. J. Bulthuis, J. Möller, H.J. Loesch, J. Phys. Chem. 101, 7684 (1997).

13. H.J. Loesch, A. Remscheid, J. Chem. Phys. 93, 1990 (1990).

14. B. Friedrich, H.G. Rubahn, N. Sathyamurthy, Phys. Rev. Lett. 69, 2487 (1992).

15. A. Slenczka, B. Friedrich, D. Herschbach, Chem. Phys. Lett. 224, 238 (1994).
16. B. Friedrich, D. Herschbach, Nature 353, 412 (1991).

17. D. Normand, L.A. Lompré, C. Cornaggia, J. Phys. B 25, L497 (1992).

18. B. Friedrich, D. Herschbach, Phys. Rev. Lett. 74, 4623 (1995).

19. B. Friedrich, D. Herschbach, J. Phys. Chem. 99, 15686 (1995).

20. G. Hadinger, M. Aubert-Frécon, G. Hadinger, Eur. Phys. J. D 4, 63 (1998).

21. F. Engelke, G. Ennen, K.H. Meiwes, Chem. Phys. 66, 391 (1982).

22. M.J. Frisch, G.W. Trucks, H.B. Schlegel, P.M.W. Gill, B.G. Johnson, M.A. Robb, J.R. Cheeseman, T. Keith, G.A. Petersson, J.A. Montgomery, K. Raghavachari, M.A. Al-Laham, V.G. Zakrzewski, J.V. Ortiz, J.B. Foresman, C.Y. Peng, P.Y. Ayala, W. Chen, M.W. Wong, J.L. Andres, E.S. Replogle, R. Gomperts, R.L. Martin, D.J. Fox, J.S. Binkley, D.J. DeFrees, J. Baker, J.P. Stewart, M. Head-Gordon, C. Gonzales, J.A. Pople, GAUSSIAN94, Pittsburgh, 1995.

23. J.P. Perdew, Y. Wang, Phys. Rev. B 45, 13244 (1992).

24. A.J. Sadlej, M. Urban, J. Mol. Struct. Theochem. 80, 234 (1991). 